\title{
How to Anticipate the Assessment of the Public Health Benefit of New Medicines?
}

\author{
Jacques Massol ${ }^{1}$, Alain Puech ${ }^{2}$, Jean-Pierre Boissel ${ }^{3}$ and the participants in Round Table $N^{\circ}$ 7, Giens XXII* \\ 1 Phisquare Institute, The Foundation Transplantation, Besançon, France, and Department of Therapeutics, \\ University of Franche-Comté, Besançon, France \\ 2 Direction of Clinical Development, Sanofi-Aventis, Gentilly, France \\ 3 Department of Clinical Pharmacology EA643, Faculty RTH Laennec, Lyon, France
}

Text received 30 September 2007; accepted 5 October 2007

\section{Keywords:} new medicine; public health; benefit; impact; assessment; reimbursement; anticipation

\begin{abstract}
The Public Health Benefit (PHB) of new medicines is a recent and French-specific criterion (October 1999 decree) which is often only partially documented in the transparency files due to a lack of timely information. At the time of the first reimbursement application for a new medicine to the "Transparency Committee", the file is exclusively based on data from randomised clinical trials. These data are generated from a global clinical development plan which was designed a long time before the new medicine's submission for reimbursement. And this plan does not systematically provide the data needed to assess the PHB. Thus, one easily understands the difficulty to anticipate and document this recent French criterion. In France, the PHB is both one of the necessary criteria for the reimbursement submission and an indicator for the national health policy management. Its assessment also helps to identify the needs and objectives of the post-registration studies (nowadays in the scope of responsibilities of the "Drug Economics Committee"). The assessment of the PHB criterion is carried through after the marketing authorization process and is an addition to it.

To understand how to anticipate the assessment of the new medicines' PHB, one needs to consider how it differs from the preliminary step of the marketing authorization process. Whereas the evaluation for marketing authorization seeks to determine if the new medicine could be useful in a specific indication, the PHB assessment aims at quantifying the therapeutic benefit in a population, taking into account the reference treatments in this population. A new medicine receives a marketing authorization based on the data of the registration file which provides information on the clinical benefit of the new medicine in the populations of the trials and in the context of the trials. On the other side, the PHB looks at the effects of the new medicine at the scale of the general population, in real practice. The PHB components of a new medicine at first submission are the expected response of this new medicine to a public health need, the expected benefit on the health status of the population and ultimately the expected impact on the health care system. The benefit of a new medicine on the health status of a population is based on public health criteria which can be morbi-mortality or quality of life criteria. However, few registration files contain these public health criteria from the beginning and the predictive value of the surrogate criteria used in the trials is not always precisely assessed. It is, thus, difficult to quantify the expected benefit on these public health criteria.

Moreover, the data that enable to quantify the new medicine's effects according to the various characteristics of the target population, are rarely available. Similarly, the French population epidemiological data related to the indication of the new medicine are often not available at the time of the assessment. Therefore it is difficult to evaluate the expected number of events that could be avoided if the new medicine reached the market. The authors suggest to adapt the clinical development plan for a better documentation of the PHB. They specifically recommend to integrate to the judgment criteria (endpoints) of the trials, criteria that are relevant in terms of public health, and to check for a good heterogeneity of the trial populations. They also suggest to start early enough collecting reliable national epidemiological data and the necessary elements for the assessment of the transposability of the trial results to the French population (ability to target the patients to be treated, adaptation of the healthcare system...). About the epidemiological data, the authors consider that the needs are covered in various ways depending on the diseases. To meet the needs of evaluation of the new medicines' target populations in specific indications, they recommend to use ad hoc studies as much as needed. In addition, epidemiological studies designed for market purpose with an acceptable methodology should not be systematically rejected but deserve to be presented.
\end{abstract}

* For a list of participants, please see the end of the article. 
To be able to assess the importance of the expected theoretical benefit of a new medicine in a population, the authors underline the necessity to have access to study results with criteria related to this objective. They suggest to first define and list the criteria by disease. Regarding the representativity of the populations, it comes out that it would be advisable, but unrealistic to include in trials a population $100 \%$ representative of the population to be treated. Therefore the effect of the new medicine must be modelised (the "effect model") to be evaluated in the general population. Yet to obtain a reliable effect model, the study population must be sufficiently heterogeneous, which legitimates the demand to ensure a good population heterogeneity at the time of decision-making about trials methodology. When the criteria assessed during the development plan does not correspond to the PHB criteria, the only way to evaluate the number of events related to the PHB criterion is, again, to use modelisation. However, modelisation is only possible when the scientific literature has established a reliable correlation between the two types of criteria. In this case, the new model should be applied to a French target population to assess the expected benefit.

As a conclusion, the possibilities to estimate the expected benefit of a new medicine on the health status of a specific population are currently limited. These limitations are regrettable because such an estimate is feasible without disrupting the development plans. The authors' general recommendations to update the development plans seem especially appropriate as the additions should not only be beneficial to France but to all the health authorities who would wish to assess the expected benefit of a new medicine on their territories. Anticipating the lack of clinical and epidemiological data and the lack of data that enable to evaluate the transposability of the trials results to real clinical practice is a sine qua none condition to improve the PHB assessment. The anticipation of these needs should be planned early enough by the pharmaceutical companies which could in this purpose meet the health authorities and the heads of the French public health policy in a consultation. Finally, because of the PHB's universal dimension, it is suggested that the necessary actions and publications be initiated so that the PHB can be acknowledged at the European level.

\section{Introduction}

The French public health authorities, seeking the right decisions in their evaluation of new medicines, increasingly need to a priori evaluate their next decisions by anticipating and quantifying as precisely as possible the expected consequences of these decisions. Similarly, there is a growing necessity to try and measure the a posteriori impact of the decisions involving the public health and resources. Such an approach in the evaluation of new medicines has become a rightful demand in a society willing to be informed of the reasons underlying the decisions taken in its name.

Both the marketing of new medicines and their admittance to the reimbursement scheme (the only real step resulting in the distribution of the new medicine at population scale) are prime public health actions. Thus both actions are imperative targets in the process of new medicines' evaluation. In France, a new medicine's admittance to the reimbursement scheme is based on collective contribution and results in a broad distribution of the new medicine. The evaluation process laying the conditions of admittance to the reimbursement scheme is led by the "Transparency Committee" (TC, in French: Commission de la Transparence), a scientific and independent committee reporting to the French High Authority of Health (in French: Haute Autorité de Santé).

The assessment criteria and rules underlying the TC's decisions are detailed in the so-called "transparency decree" dated October $1999^{[1]}$ and explicited in the literature. ${ }^{[2]}$ Two specific parameters are defined in this decree: the "actual clinical benefit" (in French: service médical rendu) of a new medicine to the pop- ulation, which acts as a guide in the reimbursement decision process, and the improvement of the actual clinical benefit of the new medicine compared to the available therapies (in French: amélioration du service médical rendu) which is at the basis of the negotiation of the new medicine's selling price and used in the estimation of the new medecine's target population, and consequently of the expected importance of the reimbursement.

Another criterion, the "public health benefit" (PHB, in French: Intérêt de Santé Publique) of a new medicine was initially created in order to grant admittance to the reimbursement scheme to new medicines for which the reimbursement could not have been justified by scientific data. Therefore, the PHB was once a component of the actual clinical benefit. This is now a different notion following the 2000-2002 round table led by the French General Direction of Health (in French: Direction Générale de la Santé), a section at the French Ministry of Health in charge of coordinating and monitoring the public health policy in France. ${ }^{[3]}$ It is now considered that a new medicine scores a PHB on the basis of the benefit rendered by that new medicine to society, either because that new medicine helps to significantly and globally improve the population's health status, or because that new medicine does not particularly affect the population's global health status but brings a response to a public health need, or still because the new medicine allows to spare resources that can then be used to improve the population's health status. Today an entire section in the TC's advices deals with the PHB criterion for every new medicine seeking admittance to the reimbursement scheme. Yet, in reality, the data presented in the submission files generally offer 
little chance for a precise assessment of the new medicines' PHB score. Collecting the missing information means extending the duration of the assessment process, which is not an option, especially in the case of supposedly innovating products. Therefore, it seems not only preferable but also essential to anticipate the information needed in order to correctly and readily assess a new medicine's PHB during its evaluation process by the TC.

After a first description of the components of the PHB criterion and detailing the data necessary for the assessment of these components, the authors will present the data that, to date and in their opinion, are the most frequently lacking and the most restricting in the assessment process of the aforementioned components. They will then suggest a number of actions and recommendations that may help with the anticipation and improvement of the assessment of new medicines' PHB.

\section{The components of the PHB and the data needed for their assessment}

The components of a new medicine's PHB are that new medicine's expected and rendered benefit to population health, its response to an existing public health need, and its potential impact on the health care system. To be able to assess these components, it is necessary to extend the assessment scope beyond the data presented in clinical and therapeutic trials which only show the new medicine's benefit to a limited population. It is essential to investigate the new medicine's benefit at the scale of the global population, in real practice and taking into account the best treatments in use.

\section{1. New medicines' benefit to the population health status}

The benefit of a new medicine to the population health status is the impact on the health rendered by or expected from that new medicine, either before the new medicine's distribution (on first admittance to the reimbursement scheme) or some time after the new medicine's admittance to the reimbursement scheme. The benefit to the population health status results from the new medicine's effect on the treated, target population (i.e. direct impact), and sometimes on the untreated population (i.e. indirect impact). A direct impact is theoretically the result of the new medicine's benefits and adverse effects on the treated, target population. This chapter is restricted to the assessment of the expected direct impact (or benefit) on the target population.

The information needed to assess a new medicine's expected direct benefit on the target population is the kind of data which enables to quantify the new medicine's benefit to the health status of a target population in France (figure 1).

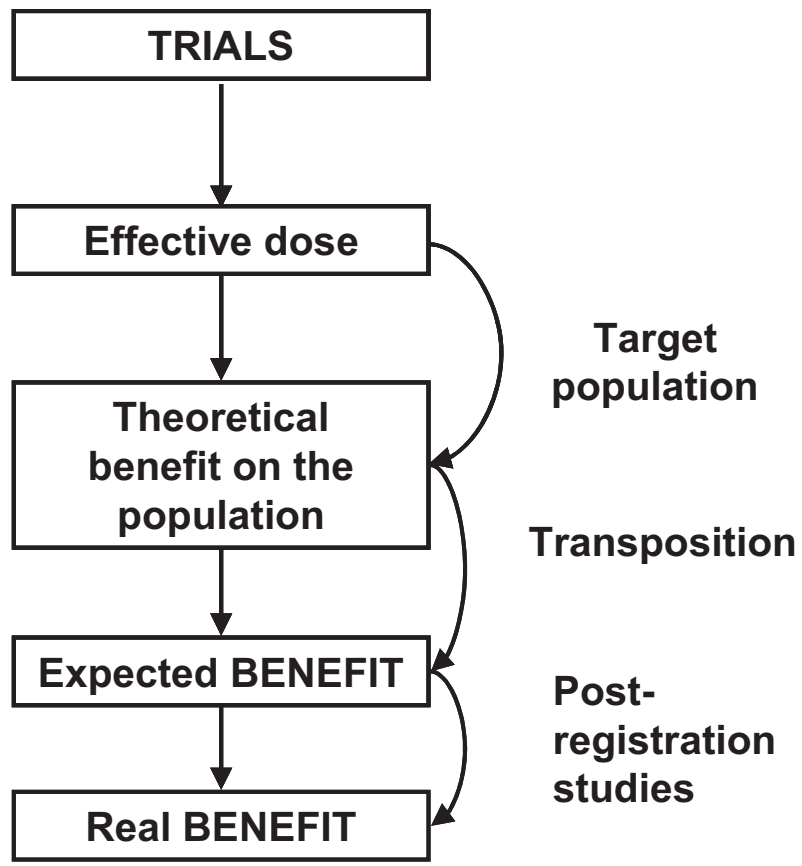

Fig. 1. Process of the Public Health Benefit assessment.

These data are of two types: (i) the data which enable to assess the new medicine's theoretical benefit, based on the (ideal?) hypothesis that the new medicine's effect in real practice has in average the same importance on the treated population than on the population used in the clinical trials, and that the treated population is indeed the expected target population. The theoretical benefit is generally an optimum; (ii) the data which enable to convert the new medicine's theoretical benefit into the expected benefit in real practice, using the transposability elements described by the members of the Giens 2005 round table. ${ }^{[4]}$ Right now and for the close future, the transposability data are not sufficiently documented to allow a quantitative and reliable conversion of the theoretical benefit. But the transposability data are and should be more and more used as a semi-quantitative weighting factor of the theoretical benefit.

In order to assess a new medicine's theoretical benefit, it is necessary in the first place to know the effective dose of that new medicine as well as that of the new medicine's best reference speciality, since the comparisons between the new medicine and its best reference speciality should make it possible to extrapolate the new medicine's expected benefit to the health status of the French target population. Given the difficulty of a quantitative comparison of new medicines' benefit/risk ratios, ${ }^{[5]}$ the comparisons should primarily be based on the new medicines' efficacy data only. The differences in tolerance - which often are about different factors according to new medicines - are to be considered in a second time as weighting factors of the benefit. In order to assess a new 
medicine's PHB, the efficacy data should be documented on criteria that are relevant for public health, i.e. according to the case, the benefits in mortality/morbidity, the handicaps that could be avoided and/or the benefit in life quality. When the main evaluation criteria presented in the trials differ from the criteria used for PHB assessment, which is often the case, data issued from surrogate criteria may be considered for the first modelisation step, provided that those surrogate criteria are predictive of events relevant for public health. Through lack of direct comparisons between a new medicine and its best reference speciality, indirect comparisons of high methodological quality should be presented. Meta-analyses may be of interest to improve the assessment of the theoretical benefit, first because of their inherent stronger statistical power and their precise evaluation of the importance of the treatment's effects, but also due to the specific fact that metaanalyses show a close evaluation of a new medicine's expected benefit in real practice using data issued from a great range of patients. In addition, meta-analyses may also sometimes help answer issues on the PHB criteria that were not initially investigated during the trials. To be able to extrapolate a new medicine's expected benefit to the French target population, one needs to have access, in addition to the efficacy data, to French epidemiological data (i.e. size and characteristics of the target population), or else, through lack of it, to epidemiological data that may be extrapolated to the French population. All these data are necessary to be able to use modelisation, which at this level is the only available method to quantify the theoretical benefit, using for instance the "effect model". ${ }^{[6,7]}$ The resulting models must present methodological guarantees (justified and fully documented data; sensitivity analyses...) as described in the literature. As for prolonged treatments, for which data about the expected treatment duration are always lacking, a number of realistic hypotheses about the effect of the treatment over time should be presented.

The theoretical benefit should be expressed as a number of events that could be avoided, and ideally as the expected alleviation of the disease burden stated in DALYs ("Disability Adjusted Life Years" = a population-related score combining morbimortality and handicap/life quality to evaluate the severity of a disease). ${ }^{[8]}$

In order to convert the theoretical benefit into the benefit expected in real practice, transposability data are needed. Unlike all the data mentioned so far, which are of a similar nature (effective dose and epidemiological characteristics of the target population) whatever the new medicine, the data necessary to evaluate the transposability vary according to the new medicines and to the diseases. If no important difference is expected between the importance of the effect detected during the trials and the importance of the effect to be expected in real pratice in the French target population (i.e. the transposability is guaranteed), a list of justifications should be provided. When an important difference is to be expected (e.g. the study population or the trials conditions differ from real practice, the ability to identify the patients is not guaranteed, a significant non compliance to the new medicine prescription is to be expected...), data on the most significant variability factors should be presented. For instance, when the new medicine's effect is liable to a great variability depending on the way the target patients are identified, it is necessary to present a performance analysis of the method used to identify the target patients. Similarly, when the new medicine's effect, to be fully displayed, is dependent on a specific set up (e.g. fibrinolysis in cerebrovascular accidents), the benefit expected in real practice in the French target population cannot be extrapolated from the data issued from a trial that was carried out in optimum conditions. This benefit needs to be adjusted depending on the set up system, and possibly including hypotheses on its expected evolution (which part of the handicap may be prevented with fibrinolysis in the current care system of cerebrovascular accidents in France?).

\subsection{New medicines' response to a public health need}

Independently of their occurrence rate, a number of severe diseases are real public health needs due to the lack of reference treatments or because these diseases are listed as public health priorities in France. Any new medicine that may bring a response to these types of public health needs scores a PHB, even though the expected benefit of that new medicine is quantitatively low. In this case, the data should first describe the way the new medicine, in its specific indications, corresponds to a real public health need in the country. The importance of that need should be quantitatively (disease burden in DALYs) assessed. Moreover, the new medicine's expected benefit on the health status of the target population should be clearly and reliably assessed, based on criteria that are relevant in terms of public health: what level of reliability can be expected from the new medicine in improving the morbidity, mortality and life quality of the patients within the target population?

\subsection{New medicines' impact on the health care system}

New medicines may spare or use resources, or still be neutral. New medicines may score a PHB if they spare resources significantly and independently of their benefit on the population health status. In case a new medicine needs a specific set up or when its effect appears to be closely depending on the health care system, it is important that the Health Authorities be warned as early as possible so that the system can be organised in the best way before the new medicine reaches the market. The data should document the 
link between the new medicine's effect and the health care system, and also detail and quantify the expected savings in resources.

\section{The information lacking in the files submitted to the Transparency Committee}

In the submission files of new medicines seeking a first admittance to the reimbursement scheme, the clinical data available are mainly the data presented in the marketing authorization file, with the addition of data to evaluate the French target population. The marketing authorization file follows a global development plan designed early before the reimbursement submission process, and therefore does not systematically present all the data needed for PHB assessment.

A retrospective review of the Transparency Committee files from May 2004 to October 2005 showed that a low expected PHB was assigned to $35 \%$ of the new medicines, an average value to $3 \%$ of the new medecines, and none to $62 \%$ of the new medecines. Only $38 \%$ of the files presented information that made it possible to assess the new medicines' $\mathrm{PHB}$, the lack of data resulting in an under-evaluation or absence of PHB.

First on the list of the elements that accounted for a low expected PHB or the absence of it, was the insufficient amount of data to correctly quantify the new medicines' benefit on morbimortality and life quality:

- few reimbursement submission files presented straightaway data on criteria that are relevant for public health, and the predictive value of the surrogate criteria used in the trials could not be fully assessed to quantify their expected benefit;

- the data which enable to quantify the new medicine's effect according to the various characteristics of the target population were rarely available;

- no direct or indirect comparisons with the best reference treatments or with the usual reference drugs were available in many files, whereas the diseases involved had an identified reference treatment acknowledged by the marketing authorization or by clinical practice recommendations;

- the target population was very rarely precisely identified due to the lack of epidemiological data for a number of the diseases, or to the lack of access to the existing epidemiological data for others;

- when it was possible to quantify the expected benefit in efficacy with suitable epidemiological data, a model of the expected benefit as the number of events that could be avoided (i.e. the importance of the benefit to be expected from the new medicine based on the efficacy data in the study population of the clinical trials, extrapolated to the theoretical target population) was very rarely available;
- finally and very frequently, through lack of data about the transposability of the trials results into real practice, and anticipating the possibility of a lower effect in real practice, the evaluators considered that the transposability was not guaranteed and therefore had to lower the importance of the benefit, and consequently the final value of the expected PHB.

Publicizing a standard reference submission file as well as the "PHB group" 's review on new medicines' evaluation principles and methods (internal document currently under review) has considerably improved the situation. In particular, the data provided on the target population using the available epidemiological data, as well as the information provided on the notion of public health need ${ }^{[9,10]}$ have been developed. Nevertheless these elements are still very much insufficiently documented. Also the quantification of the burden of disease in France stated in DALYs is still insufficiently documented; but it is not the case for the data provided on the severity of the disease at individual level. In addition, quantifiying the new medicine's expected benefit on morbi-mortality and life quality, compared to the reference treatments, is still not possible due to the lack of data that will only be accessible through an anticipation approach. Finally, the information supporting the assumption of the new medicines' response to an existing public health need and regarding the new medicines' impact on the health care system is still too scarce.

\section{Recommendations for a better anticipation of the PHB assessment}

As the data presented to date in the submission files to the Transparency Committee is insufficient to correctly assess the new medicines' PHB and in particular to quantify their expected benefit, the authors suggest a number of actions below with the aim of having access to all the necessary data at the right time of submission to the Transparency Committee. A number of these recommendations are intended for the public authorities and associated agencies, the rest is intended for the pharmaceutical industry. The main point is to plan an early consultation between the public authorities and the pharmaceutical companies in order to define in advance and anticipate the data to be provided in each file.

\section{1. Updating the new medecines' development plans}

The possibilities to assess a new medicine's expected benefit on a given population's health status are currently limited. This is all the more regrettable since assessing a new medicine's expected benefit is feasible without disrupting or extending the duration of the new medicines' development plans. A realistic modification of the new medicines' development plans would consist in including 
criteria that are relevant in terms of public health (the said "PHB criteria") in the list of the criteria investigated during the trials, and also selecting a study population with a good heterogenity so that the population used in the trials is most similar to the population that is to receive the new medicine. Moreover, it is recommended not to leave out the subgroups which are thought to benefit the most from the new medicine (e.g. stratification).

A preliminary task should be the definition of the PHB criteria for each disease. Even though the PHB criteria fail to be considered as essential criteria for the submission process, it is important that the PHB criteria be viewed and, whenever possible, included as secondary efficacy criteria during the clinical trials before filing for marketing authorization. At the minimum, the clinical trials should focus on events relevant for public health. The authors recommend that a preliminary work defines more precisely the relevant events to be considered for each disease. This global recommendation to update the new medicines' development plans appears all the more legitimate since the requested modifications should not only benefit France, but also the health authorities in all countries willing to assess a new medicine's expected benefit on their territories.

\subsection{Improving the possibilities of accessing epidemiological data}

The lack or the little amount of epidemiological data is one of the most restricting limits to the definition of a new medicine's target population and to the quantification of it expected benefit. The availability of epidemiological data in France varies depending on the diseases. The authors' recommendation is to keep on complementing the existing inventory of epidemiological data (initiated by the French Health and Medical Research Institute (in French: Institut National de la Santé et de la Recherche médicale, INSERM), to facilitate the collection of new and useful epidemiological data, to improve the access to the existing epidemiological data (lists and type of access to databases and cohorts), and to use $a d$ hoc studies as often as necessary with their objectives described as soon as the new medicine's indication is identified. Also, the epidemiological studies designed for market purpose, provided that their methodology is acceptable, should not automatically be rejected and ought to be presented, or possibly modified with the objective of compliance with the Good Epidemiological Practice.

\subsection{Improving the receivability of modelisation as a reference method for the assessment of the expected theoretical benefit}

The only way to quantitatively assess a new medicine's expected benefit on a global population is modelisation. A prerequi- site for modelisation is to have access to the efficacy data based on the PHB criteria (morbi-mortality, life quality). The modelisation method may be for instance the "effect model". ${ }^{[6,7]}$ However the study population needs to show a good heterogeneity in order to ensure the reliability of the effect model, hence the authors' recommendation to be particularly careful of the population heterogeneity and of the efficacy criteria at the time of decision-making about the trial's methodology.

Regarding the efficacy data though, it would be unrealistic to expect all data on the PHB criterion to be available at a new medicine's first submission for reimbursement. When the criterion evaluated during new medicine development is a surrogate criterion, the only possibility to evaluate the number of events related to the PHB criterion is a two-step modelisation. However the first modelisation step is only feasible if a reliable correlation has been shown in scientific bibliography between the abovementioned surrogate criterion and the PHB criterion. If this is the case, this new model should be used on a French target population to assess the expected benefit.

To improve the receivability of modelisation as a reference method for the assessment of the expected theoretical benefit, the authors recommend that a methodological guide is prepared and distributed and that examples of models are made available as a help for PHB assessment. The evaluation and investigation of these models should be managed by a third party to prevent any potential bias (figure 2).

\subsection{A better definition of public health needs as regards new medicines}

In spite of all the public health policy's objectives, indicators and programmes, the priorities in public health are particularly badly covered therapeutically, and, with the exception of specific cases (uncommon diseases, drugs' pediatric versions...), are not clearly detailed. Hence the suggestion to elaborate a methodology to identify the important public health needs in new medicines that are not at all or not sufficiently covered. Also the authors suggest to list ten diseases for which the needs in research and new medicine development may be considered as priorities.

\subsection{Planning an early consultation with the pharmaceutical industry}

An absolute, sine qua non condition to the improvement of the PHB assessment is being able to anticipate the needs for complementary clinical and epidemiological data, for data that enable to evaluate the level of transposability of the trials results to real clinical practice, or for data that enable to assess the new 


\section{Several levels of investigation}

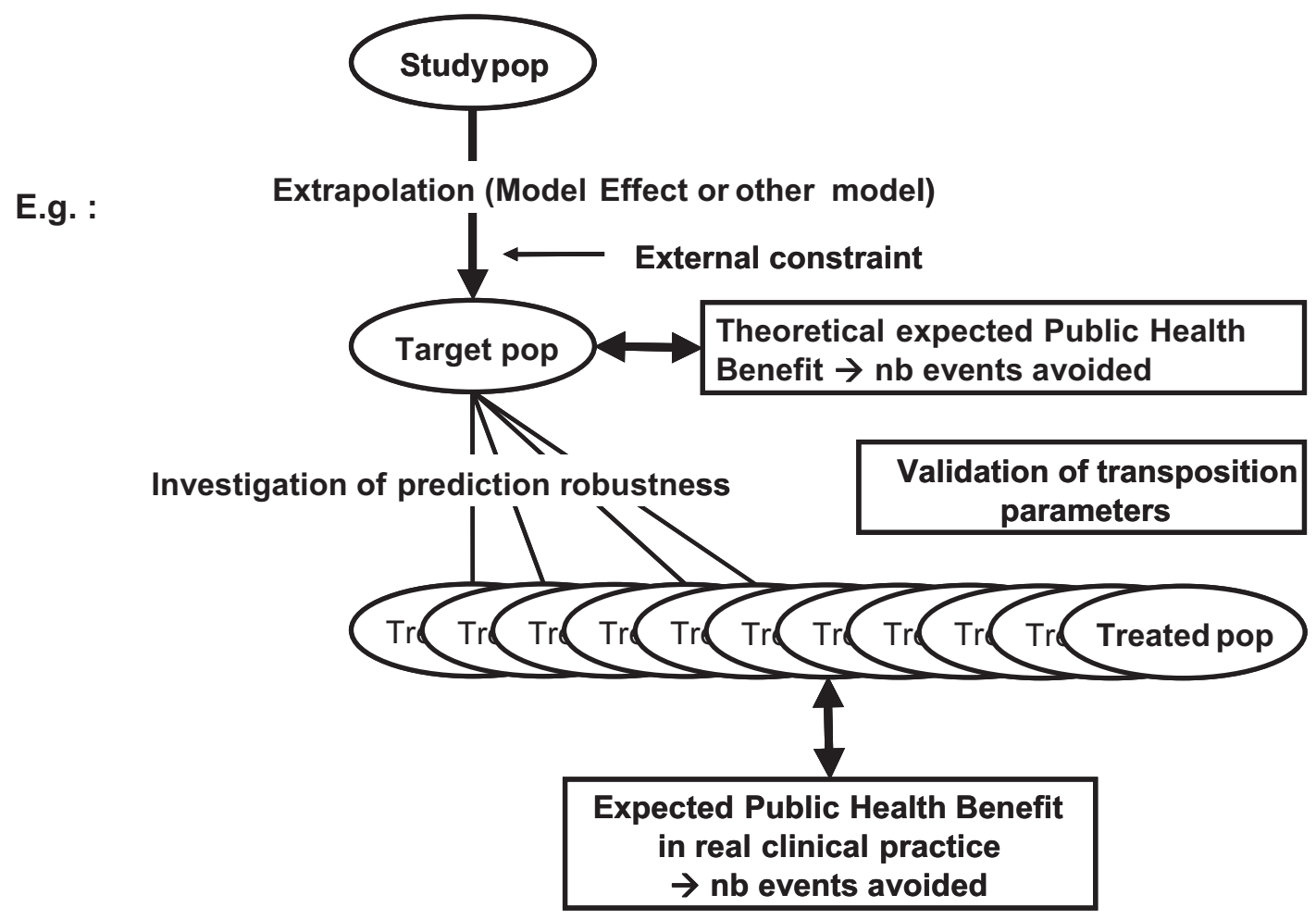

Fig. 2. The use of modelisation. pop : population, $\mathbf{n b}$ : number of.

medicine's impact on the health care system. A better information about the Health Authorities' requests should, to some extent, encourage a more complete and unsolicited documentation of PHB data in the new medicines' submission files ( $c f$. supra). Nevertheless the development plans should be adapted in order to answer public health issues that are not currently dealt with in the marketing authorization files, although the pharmaceutical companies will be reluctant to invest in unnecessary or worthless new steps. Therefore for every file, a consultation should be programmed between the pharmaceutical companies, the heads of the public health authorities and the evaluators for an early identification of the data to be provided for PHB assessment. Such a consultation, which could take place towards the end of development phase II, would enable pharmaceutical companies: to evaluate to what extent the scheduled development plan could make it possible for the PHB data to be collected on time; to define the ad hoc studies necessary to identify the target population; to list the data necessary to modelise the theoretical expected benefit; to ensure the transposability of the trials results to the French target population (identification of patients, adaptation of the health care system...); if necessary, to document the new medicine's impact on the health care system. At this stage also, the need for post-registration studies could be partially anticipated.

A preliminary work should be carried out to evaluate the feasibility of such consultations, the corresponding human resources and work load, the conditional participation of the pharmaceutical companies, the confidentiality rules and the responsibilities of all actors during these consultations. Since this type of consultation would have predictable consequences both on the marketing authorization files and the reimbursement submission files, it would be useful and logical to have both the actors in charge of new medicine evaluation and the public health authorities attending this kind of consultation.

\subsection{The value of new medicines according to their PHB}

In addition to the fact that the files submitted to the TC hardly enable the TC to make a reliable assessment of new medicines' PHB, there is currently no difference in the way new medicines with a PHB and new medicines with no PHB are considered. Even though a high PHB is implicitly taken into account for new medicines' pricing evaluation by the "Drug Economics 
Committee" (in French: Comité d'Évaluation des Produits de Santé), the lack of explicit instructions about the value of new medicines according to their PHB is a weak incentive for pharmaceutical companies to provide a better documentation on PHB. In order to encourage the pharmaceutical companies to put more effort in anticipating and collecting the necessary data, it would be important and adequate that the next decree first grants a more consistent dimension to the PHB criterion, and secondly details the way the PHB criterion could be better perceived.

\section{Conclusion}

The assessment of a new medicine's PHB is a natural and rightful concern of the public health authorities in a country that wishes to build a public health policy based on factual data, to anticipate and to evaluate the consequences of its decisions, and to use its collective resources at best. To get to this new stage in new medicine evaluation, the pharmaceutical industry needs clear and transparent guidelines and instructions.

The data presented to date in the marketing authorization files are insufficient to assess the new medicines' PHB. And when the time of the PHB assessment comes, it is too late to collect the missing data. Therefore it is absolutely essential to anticipate as early as possible in the process, i.e. from the end of development phase II, the necessity to provide the complementary data. The pharmaceutical companies must indeed consider the issue of the lacks and the requirements in their files from this early stage on, if the situation is to be improved. Also, the health authorities should launch an early discussion with the pharmaceutical industry about the need of anticipation and in order to check that all data necessary to quantify the PHB are included in the development plans, suggesting adaptations if need be.

Prerequisite to this stage, it would be necessary to draw up a more accurate inventory of the data lacking in the files submitted to date to the TC for PHB assessment, to initiate a feasibility study and issue a number of rules about setting up consultations between the pharmaceutical companies and the health authorities, and to develop the modelisation methodology used to assess the new medicines' expected benefit.

Finally, the notion of the PHB criterion which is specific to France and under-used to appreciate the value of new medicines should be extended to other countries and used more extensively. Without having to resort to more resources, and since the data presented to date in the files submitted to the TC are not optimally exploited to assess the expected $\mathrm{PHB}$, the authors recommend that the notion of PHB and the process of PHB assessment be more broadly publicized and explicited, both nationally and internationally. Given the universal dimension of the PHB criterion, it would be useful to undertake the adequate actions and publications in order for the PHB criterion to be acknowleged at least first at the European level. Finally a modification of the "transparency decree" in France should explicit and acknowledge the importance of the PHB criterion.

Acknowledgements. Thanks to Claire Latruffe (Fondation Transplantation) for her assistance in the translation and finalization of the article.

\section{Participants}

A. Alperovitch (INSERM U708, Hôpital Salpêtrière, Paris), M. Bamberger (BMS, Rueil Malmaison), B. Begaud (CHU Pellegrin, Bordeaux), O. Blin (Hôpital Sainte Marguerite, Marseille), P. Blin (Paris Biotech, Paris), G. Breart (INSERM U149, Villejuif), C. Brun Strang (Novartis Pharma, Rueil Malmaison), M. Buyse (Institut National du Cancer, Boulogne Billancourt), A. Castot (AFSSAPS, Saint-Denis), M. Chauvenet (Aremis Consultants, Neuilly), A. Chicoye (Aremis Consultants, Neuilly), N. David (Direction Générale de la Santé, Paris), F. De Bels (Haute Autorité de Santé, Saint-Denis la Plaine), R. De Sahb Berkovitch (Roche, Neuilly-sur-Seine), E. Dohin (Merck Sharpe \& Dohme Chibret, Paris), F. Fagnani (Cemka, Bourg la Reine), B. Falissard (INSERM U669, Hôpital Cochin, Paris), C. Gastaldi-Menager (Direction Générale de la Santé, SaintDenis), I. Giri (Access, Verrières le Buisson), M. Haim (Merck Sharp \& Dohme, Paris) J.M. Joubert (Schwarzpharma, Paris), M. Lapeyre-Mestre (CHU Purpan, Toulouse), C. Lassale (LEEM, Paris), I. Marchant Ramirez (UMR CNRS 5558, Bron), F. Meyer (Haute Autorité de Santé, Saint-Denis la Plaine), J. Micallef (Hôpital Sainte Marguerite, Marseille), M. Mollimard (CHU Pellegrin, Bordeaux), T. Moreau-Defarges (Janssen-Cilag, Issy les Moulineaux), L. Pazart (CHU Saint-Jacques, Besancon) L. Perret (Servier, Neuilly-sur-Seine), M. Pigeon (CNAM, Paris), C. Rumeau Pichon (Haute Autorité de Santé, Saint-Denis la Plaine), Ph. Tcheng (Sanofi-Aventis, Paris), M. Woler (Janssen-Cilag, Issy les Moulineaux), L. Zanetti (AFSSAPS, Saint-Denis), M. Zylberman (Lilly France, Suresnes).

\section{References}

1. Décret "de la Transparence" n 99-915, 27 October 1999 http://www . legifrance.gouv. fr/WAspad/UnTexteDeJorf? numjo=MESS9923357D

2. Massol J, Le Jeunne C. Comment évaluer l'intérêt des médicaments ? Presse Med 2007; 36(3 Pt 2): 505-11

3. Direction Générale de la Santé. "L'évaluation de l'impact d'un médicament sur la santé des populations et la santé publique". January 2002 http://www.rees-france.com/IMG/pdf/DOC_evaluation_dgs.pdf

4. Massol J, Zylberman M, Goehrs JM and the participants to the Round Table $\mathrm{N}^{\circ} 1$, Giens XXI. Use of the Foreign Studies: Transposition of the Results, 
Prediction of the Therapeutic Effects in the French Population, Modelling of the Public Health Interest. Therapie 2006; 61(6): 491-9

5. European Medicines Agency London, Committee for Medicinal Products for Human Use. "Report of the CHMP working group on benefit-risk assessment models and methods" (ref. EMEA/CHMP/15404/2007). 19 January 2007 http:// WwW . emea . europa. eu/pdfs/human/brmethods/1540407en.pdf

6. Boissel JP, Collet JP, Lievre M, et al. An effect model for the assessment of drug benefit : Example of antiarrhythmic drugs in postmyocardial infarction patients. J Cardiovasc Pharm 1993; 22: 356-63

7. Boissel JP, Cucherat M, Nony P, et al. New insights on the relation between untreated and treated outcomes for a given therapy. Accepted for publication in J Clin Epidemiol
8. World Health Organization's Global Burden of Disease Project http://www . who.int/healthinfo/bodabout/en/index.html

9. Direction Générale de la Santé, GTNDO's 2003 report http:// www.sante.gouv.fr/htm/dossiers/losp/rapport_integral.pdf

10. Loi $\mathrm{n}^{\circ}$ 2004-806, 9 August 2004 on France's "Public Health Policy" http: //www . legifrance.gouv. fr/WAspad/UnTexteDeJorf? numjo=SANX0300055L

Correspondence and offprints: Jacques Massol, Centre Hospitalier Universitaire Saint-Jacques, Hôtel Montmartin, 25030 Besançon, France.

E-mail: jacques.massol@univ-fcomte.fr 